\title{
Redox-dependent and independent mechanisms of selective pro- and anti-apoptotic activity of Georgian legumes crops extracts on Jurkat and MDCK cells
}

\author{
Sanikidze T.V. ${ }^{1,2,4}$, Chkhikvishvili I.D. ${ }^{2}$, Maminaishvili T.L. ${ }^{3}$, Kipiani Nana V. ${ }^{1}$, Enukidze \\ M.G. ${ }^{2}$, Machavariani M.G. ${ }^{2}$, Shekiladze E.R. ${ }^{1}$, Ormotsadze G.L. ${ }^{1,3,4}$
}

${ }^{1}$ Tbilisi State Medical University, ${ }^{2} \mathrm{~V}$. Bakhutashvili Institute of Medical Biotechnology of Tbilisi State Medical University, ${ }^{3}$ Davit Aghmashenebeli University of Georgia, ${ }^{4}$ Ivane Beritashvili Center of Experimental Biomedicine

Corresponding author: Tamar V. Sanikidze, PhD, ScD, professor, Department of Physics, Biophysics, Biomechanics and Informative Technologies, Tbilisi State Medical University, Vaja Pshavela av., 33, Tbilisi, 0186, Georgia.

Submission Date: March $10^{\text {th }}$, 2019. Acceptance Date: May $28^{\text {th }}$, 2019. Publication Date: May $30^{\text {st }}, 2019$.

Citation: Sanikidze T.V., Chkhikvishvili I.D., Maminaishvili T.L., Kipiani Nana V., Enukidze M.G., Machavariani M.G., Shekiladze E.R., Ormotsadze G.L. Redox-dependent and independent mechanisms of selective pro- and anti-apoptotic activity of Georgian legumes crops extracts on Jurkat and MDCK cells. Functional Foods in Health and Disease 2019; 9(5): 357-370. DOI: https://doi.org/10.31989/ffhd.v9i5.602

\section{ABSTRACT}

Background: Possibilities of pharmacological regulation of apoptosis play an important role in the treatment of different diseases. Polyphenol-rich plant extracts as well as isolated polyphenols can regulate cell apoptosis primarily through intrinsic and extrinsic mechanisms of action and are the most intriguing and studied class of compounds that can be therapeutics for a wide range of common diseases, including cancer. Polyphenols are well known as powerful antioxidants, and their action is also associated with pro-apoptotic function in various types of tumor cells.

The purpose of this study was to establish the anti-and pro-apoptotic activity of Georgian legume crops in model cellular systems.

Methods: Legume crop extracts (LCEs) were prepared in water-alcohol solute. Polyphenol content in the extracts was determined using the Folin-Ciocalteu method; antiradical activity (AA) according the comparative time of the $50 \%$ neutralization of 2,2-diphenyl-1-picrylhydrazyl (DPPH) cleavage.

Studies were carried out on human leukemic mature $\mathrm{T}$ cells (Jurkat) and normal epithelial MDCK cell lines. For modelling of oxidative stress, $30 \%$ hydrogen peroxide was used. LCEs were added to intact or incubated Jurkat and MDCK cells under oxidative stress conditions. Cell viability was determined by 3-(4,5-dimethyltiazol-2)-2,5-diphenyl-tetrazolium-bromide (MTT) test. Catalase 
and Superoxidedismutase (SOD) activity in cellular supernatant was measured by spectrophotometry.

Results: LCEs revealed selective pro- and antiapoptotic activity on the intact and incubated cells under oxidative stress conditions Jurkat and MDCK cells. The cytotoxic effect of LCEs on intact Jurkat and MDCK cells was independent of their AA and activity of enzymatic cellular antioxidant defense system.

The cytoprotective effect of LCEs on MDCK cells was realized through redox-dependent mechanisms and is associated both with the own AA of the extracts and with the stimulating effect of the extracts on enzymatic cellular antioxidant defense system activity, where catalase played a leading role. LCEs didn't protect Jurkat cells from oxidative stress-induced apoptosis.

Conclusion: The obtained results allow us to conclude that in cancer chemotherapy, the legume extracts might be combined with prooxidant drugs in order to protect normal cells, but not malignant ones, from their apoptosis-inducing effect. On the other hand, these extracts may protect nonmalignant tissues/organs from various apoptosis-related disorders.

Keywords: Georgian legume crop polyphenol extracts, antiradical activity, antioxidant enzymatic system, oxidative stress, pro-/antiapoptotic activity

\section{BACKGROUND}

Apoptosis is an active mechanism of programmed cell death characterized by both physiological and pathological processes in a living organism. Physiological apoptosis develops during embryogenesis, the elimination of autoreactive lymphocytes from peripheral blood, etc. Apoptosis performs the opposite function of mitosis and plays an important role in regulation the size of tissues and maintaining homeostasis in a living organism. Dysregulation of apoptosis can cause the development of age-related pathologies: activation of apoptotic death in postmitotic cells (cardiomyocytes, neurons, and chondrocytes) during aging leads to myocardial infarction, the development of neurodegenerative diseases, age-related degeneration of cartilage in the joints and intervertebral discs. Diseases associated with an increased level of apoptosis include pathologies of the blood system (aplastic anemia, thalassemia, thrombocytopenia, lymphopenia, etc.); programmed cell death caused by bacterial endo- and exotoxins is a sign of sepsis, AIDS, periodontitis, etc. Disorders of T-cell apoptosis can cause autoimmune pathologies. One of the reasons for the development of malignant tumors is a violation of the mechanisms of cellular apoptosis (the activity of $\mathrm{p} 53$ and $\mathrm{Bcl}-2$ proteins as well as the regulation of apoptosis intensity). Viruses (varicella, herpes) block receptor-dependent apoptosis of infected cells by synthesizing anti-apoptotic proteins of Bcl2 family. Apoptosis can be caused by external apoptogenic factors - ionizing radiation, anticancer chemotherapy drugs, and hormones used in the treatment of various diseases [1-3].

Based on the foregoing implications, the possibilities of pharmacological regulation of apoptosis play an important role in the treatment of various diseases. Polyphenol-rich plant extracts as well as isolated polyphenols can regulate cell apoptosis primarily through intrinsic and extrinsic mechanisms of action [4] and are the most intriguing and studied class of compounds that can be therapeutics for a wide range of common diseases, including cancer. 
Various signaling systems are involved in the regulation of apoptosis through reactive oxygen species (ROS) that by modifying the redox state of cells act as messengers of the proapoptotic stimulus [5]. Cellular redox balance is an important regulator of cell activity [6-13]. Polyphenols are well known as powerful antioxidants, and their action is also associated with pro-apoptotic function in various types of tumor cells [14].

The purpose of this study was to establish the anti- and pro-apoptotic activity of Georgian legume crops in model cellular systems.

Evaluation of the anti-/proapoptotic activity of legume crop extract (LCEs) (beans (Phaseolis vulgaris) "Tirkmela," "Shulavera," "Batumela-mindvris," "Udelebi," green peas (Pisum sativum), lentils (Lens Culinaris), soybean (Soybean Glicine max $(L)$ ) was performed on experimental models of MDCK cells (epithelial cells isolated from Cocker Spaniel kidney tissues) and Jurkat cells (human leukemia-transformed $\mathrm{T}$ cells isolated from the peripheral blood of a 14-year-old boy with leukemia). Jurkat cells are widely used in studies of signaling pathways that regulate the apoptotic events in tumor cells and T-cell activity.

\section{MATERIALS AND METHODS}

\section{Materials}

The beans were purchased on the local market in Tbilisi. Legume crops samples were crushed into powder. To obtain extracts of polyphenols, $30 \mathrm{ml}$ of a water-alcohol solution was added to $1 \mathrm{mg}$ of the sample and left for 3 days. The content of polyphenols in the extracts was determined by FolinCiocalteu method. The calibrate curve was performed according Gallic acid content (mg/l) and the total content of polyphenols was calculated. Antioxidant activity of the extracts was determined from the comparative time of the 50\% neutralization of 2,2-diphenyl-1-picrylhydrazyl (DPPH) cleavage (the shorter is the time of radicals neutralization, the higher is the antioxidant activity of the extract) [15].

\section{Cell culture}

The research was conducted on human leukemic mature T cells (Jurkat cells) (DSMZ-Deutshe Sammulung von Mikroorganismen und Zellkulturen (Germania)) and MDCK cell line (Lugar Laboratory, Tbilisi, Georgia). Jurkat cells was proliferated in bioactive medium RPMI 1640 (GIBSO), inactivated fetal bovine serum (FBS) (Sigma), L-glutamine (4 mM), penicillin $(100 \mathrm{un} / \mathrm{ml})$, and streptomycin $(100 \mathrm{un} / \mathrm{ml})$ containing suspension at $37^{\circ} \mathrm{C}$ in a moist, $5 \% \mathrm{CO}_{2-}$ containing atmosphere. Experiments were carried out on cell concentration $0,3-0,6 \times 10^{6}$ cells in 1 $\mathrm{mL}$ of medium. The MDCK cells were grown in Eagle's Medium (DMEM) (Mediatech, Herndon, VA) and supplemented with $5 \% \mathrm{FBS}$, penicillin $(100 \mathrm{U} / \mathrm{ml})$, and streptomycin $(100 \mathrm{U} / \mathrm{ml})$ at $37^{\circ} \mathrm{C}$ in $5 \% \mathrm{CO}_{2}$-containing atmosphere.

\section{Modelling of oxidative stress}

For modelling of oxidative stress, $30 \%$ hydrogen peroxide $\left(\mathrm{H}_{2} \mathrm{O}_{2}\right)$ (Sigma) was added to Jurkat (in dose $50 \mu \mathrm{M}$ ) [6,15] and MDCK cells (in dose $800 \mu \mathrm{M}$ ) [11] incubation suspension with subsequent incubation for $24 \mathrm{~h}$. The control group was represented by intact Jurkat and MDCK cells.

Extracts of various varieties of legume crops $(40 \mu \mathrm{l})$ were added to the intact or incubated Jurkat and MDCK cells under oxidative stress conditions. To the cells where the extract was not added, 40 $\mu 1$ of the medium (RPMI 1640, inactivated FBS, L-glutamine, penicillin, and streptomycin 
containing suspension) was added accordingly. The effect on the Jurkat and MDCK cells of a wateralcohol solution $(96 \%$ ethanol + water $(40 \mu \mathrm{l}))$ used to obtain extracts of polyphenols was also investigated.

Cell viability was determined by 3-(4,5-dimethyltiazol -2)-2,5- diphenyl tetrazolium bromide (MTT) test.

\section{The MTT test of cells proliferation activity (viability)}

Cell suspension $\left(2 \times 10^{6}\right.$ cell $\left./ \mathrm{mL}\right)$ was incubated in growth medium (PRMI-1640 $+10 \%$ calf embryonic serum (Sigma) $+1 \%$ penicillin/streptomycin) together with (or without) hydrogen peroxide and LCEs at $37^{\circ} \mathrm{C}$ in $5 \% \mathrm{CO}_{2}$ atmosphere. After the incubation period, suspension was centrifuged at 1,500 rpm for 5 minutes. MTT (3-(4,5-dimethyltiazol -2)-2,5-diphenyl tetrazolium bromide) (Sigma) solution was added to sedimented cells ( $30 \mu \mathrm{L}$ to $100 \mu \mathrm{L}$ suspension; MMT was diluted in buffer (140 mM NaCl, $5 \mathrm{mM} \mathrm{HEPES,} \mathrm{pH} \mathrm{7,4)} \mathrm{(in} \mathrm{dose:} 300 \mu \mathrm{L}$ buffer to $2.5 \mathrm{mg}$ MTT) and incubated for 4 hours at $37^{\circ} \mathrm{C}$ in $5 \% \mathrm{CO}_{2}$ conditions. After the incubation, the dilutant $(100 \mu \mathrm{l}$ dimethyl sulfoxide (DMSO)) was added to the supernatant of the cells. Absorption of the obtained suspension was measured by the spectrophotometer at wavelength $570 \mathrm{~nm}$. For the research suspension, we determined the coefficient of proliferation $\mathrm{K}$ through the formula

$$
\mathrm{K}=\mathrm{A}_{\text {trial }} / \mathrm{A}_{\text {control }}
$$

where $A_{\text {trial }}$ - absorption rate for studying solute, $A_{\text {control }}$ - absorption rate for control solute, respectively. Coefficient of apoptosis $\left(\mathrm{K}_{1}=1-\mathrm{K}\right)$ reflects the number of cells, from total number, with low proliferative activity [16].

\section{Determination of catalase and SOD activity in Jurkat and MDCK cells}

Catalase and Superoxidedismutase (SOD) activity in Jurkat and MDCK cells' supernatants was measured by the spectrophotometry with subsequent recalculation on protein concentration by the method of O.H. Lowry.

The method of the determination of catalase activity is based on the ability of hydrogen peroxide to form a colored complex with the salts of molybdenum. The principle that was used to determine the activity of SOD proceeds from the ability of the enzyme to compete with tetrazole nitro blue for superoxide anion radicals $[17,18]$. The absorption intensity of the coloring solute was measured by spectrophotometry at a wavelength of $410 \mathrm{~nm}$.

\section{Statistical analysis}

The analysis of variance (ANOVA) was used to conduct the comparative analysis of the intensity of the apoptosis and antioxidant status indicators (SOD, catalase, AA). The analysis and visualization of data was conducted by using "SPSS-12" for Windows.

\section{RESULTS AND DISCUSSION}

The study was devoted to the identification of LCEs with potential pharmacological properties and investigation of the molecular mechanisms of their action in normal and pathological conditions. As a model system for the study, epithelial MDCK and leukemia-transformed (tumor) Jurkat cells were used.

Figure 1A shows the results of a study of the polyphenols content in various LCEs and their antioxidant activity. The correlation analysis of the data shown in Figure 1B indicates the direct 
correlation between the phenols content and antiradical activity (AA) of the studied extracts $(\boldsymbol{r}=$ $0.79, \boldsymbol{p}=0.00002)$. These results indicate the important role of phenolic compounds in the mechanisms of the AA of LCEs. Although in some cases, for "Udelebi" beans, non-phenolic compounds (carotenoids, alkaloids and others) also contribute to the overall antioxidant effect of the extract.

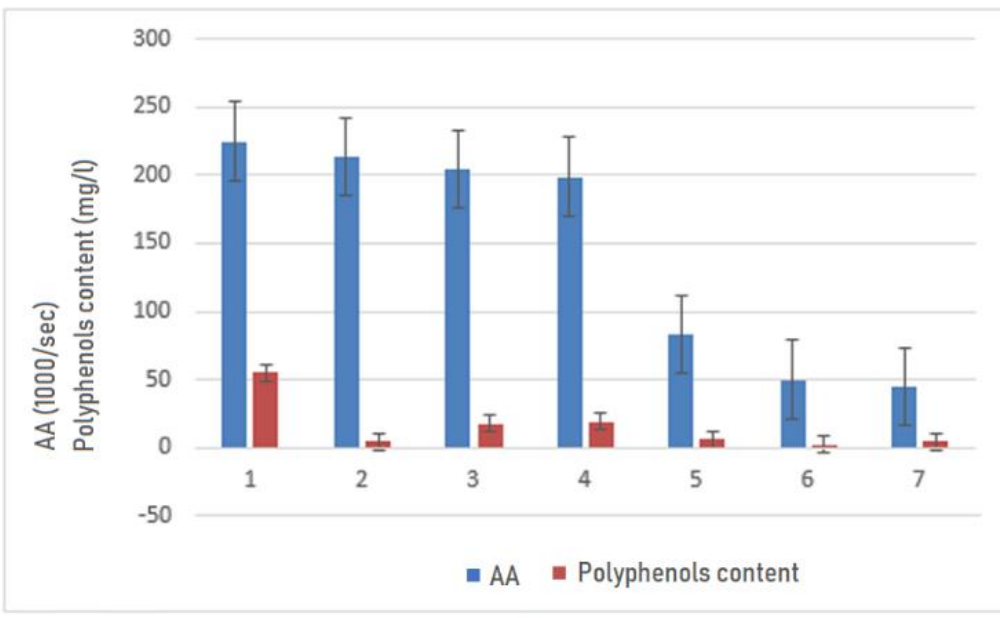

A

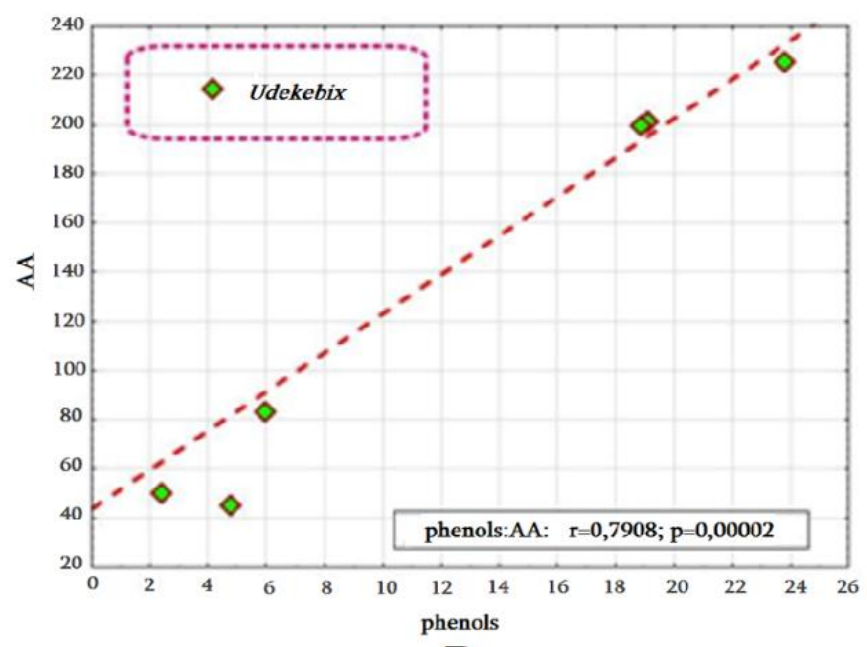

B

Figure 1. Polyphenols content and antioxidant activity of LCEs (60 ml) (A); dependence between AA and phenol content in the extracts (B) (1 - "Batumela-mindvris" beans, 2 - "Udelebi" beans, 3 "Shulavera" beans, 4 - "Tirkmela" beans, 5 - lentils, 6 - soybean, 7 - green peas)

We investigated the cytoprotective/cytotoxic (anti- and proapoptotic) effects of LCEs on the intact and incubated under oxidative stress conditions Jurkat and MDCK cells (Figures 2, 3).

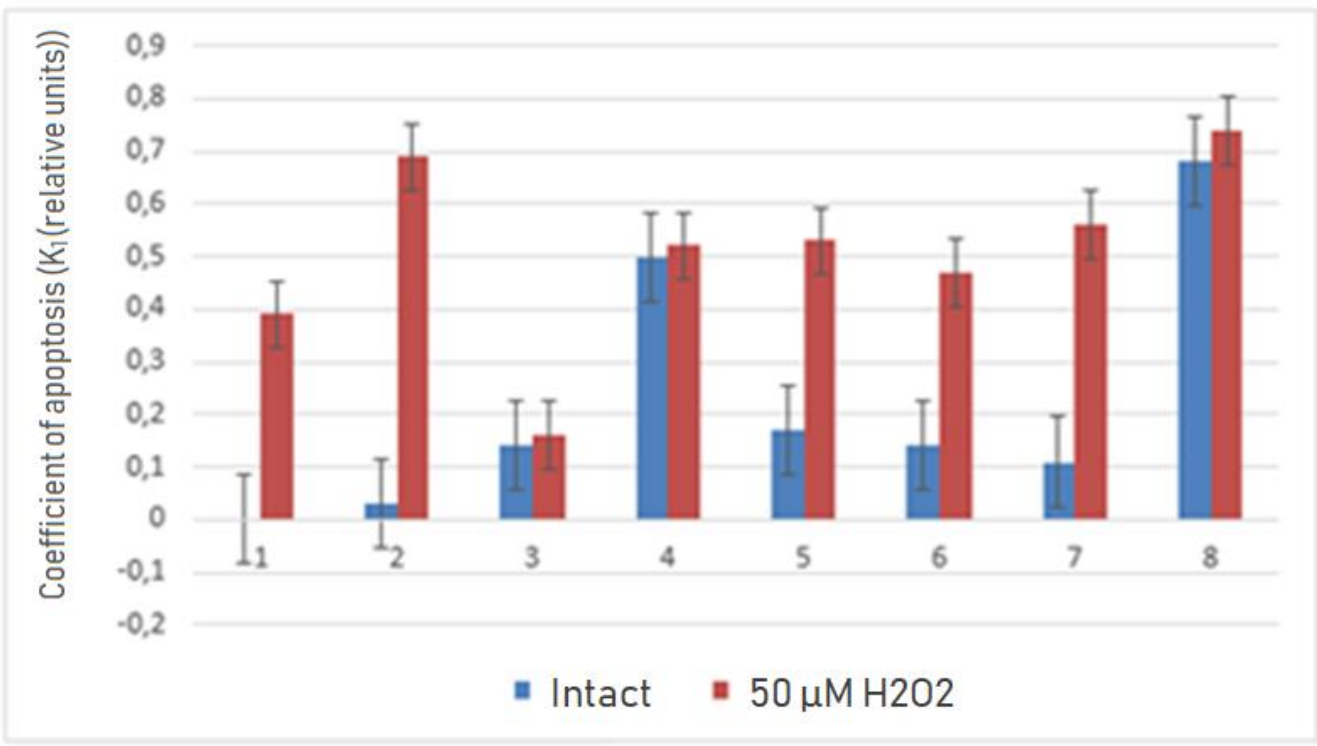

Figure 2. Coefficient of apoptosis $\left(\mathrm{K}_{1}\right)$ of Jurkat cells incubated in different conditions (1- Jurkat cells, 2 - Jurkat + beans "Tirkmela", 3 - Jurkat + "Batumela-mindvris" beans, 4 - Jurkat + beans "Udelebi”, 5 - Jurkat + beans "Shulavera", 6 - Jurkat + green peas, 7 - Jurkat + lentils, 8 - Jurkat + soybean). 
From the results presented in the diagrams 2 and 3, it follows that during the joint incubation of "Udelebi," "Shulavera," and soybean extracts with intact Jurkat and MDCK cells, the cytotoxic effect was detected in 50\% (Jurkat, MDCK), 49\% (MDCK), and 68\% (Jurkat) as well as 34\% (MDCK) of the total number of cells, respectively. The remaining extracts were relatively inert with respect to Jurkat and MDCK cells while lentils exhibited 15\% stimulating activity on the intensity of the proliferation of MDCK cells. No significant change in the value of the apoptosis $\left(\mathrm{K}_{1}\right)$ coefficient was detected when the water-alcohol solution $(96 \%$ ethanol + water $(40 \mu 1))$, used to obtain extracts of polyphenols, was added to the incubation medium of Jurkat and MDCK cells (data are not shown).

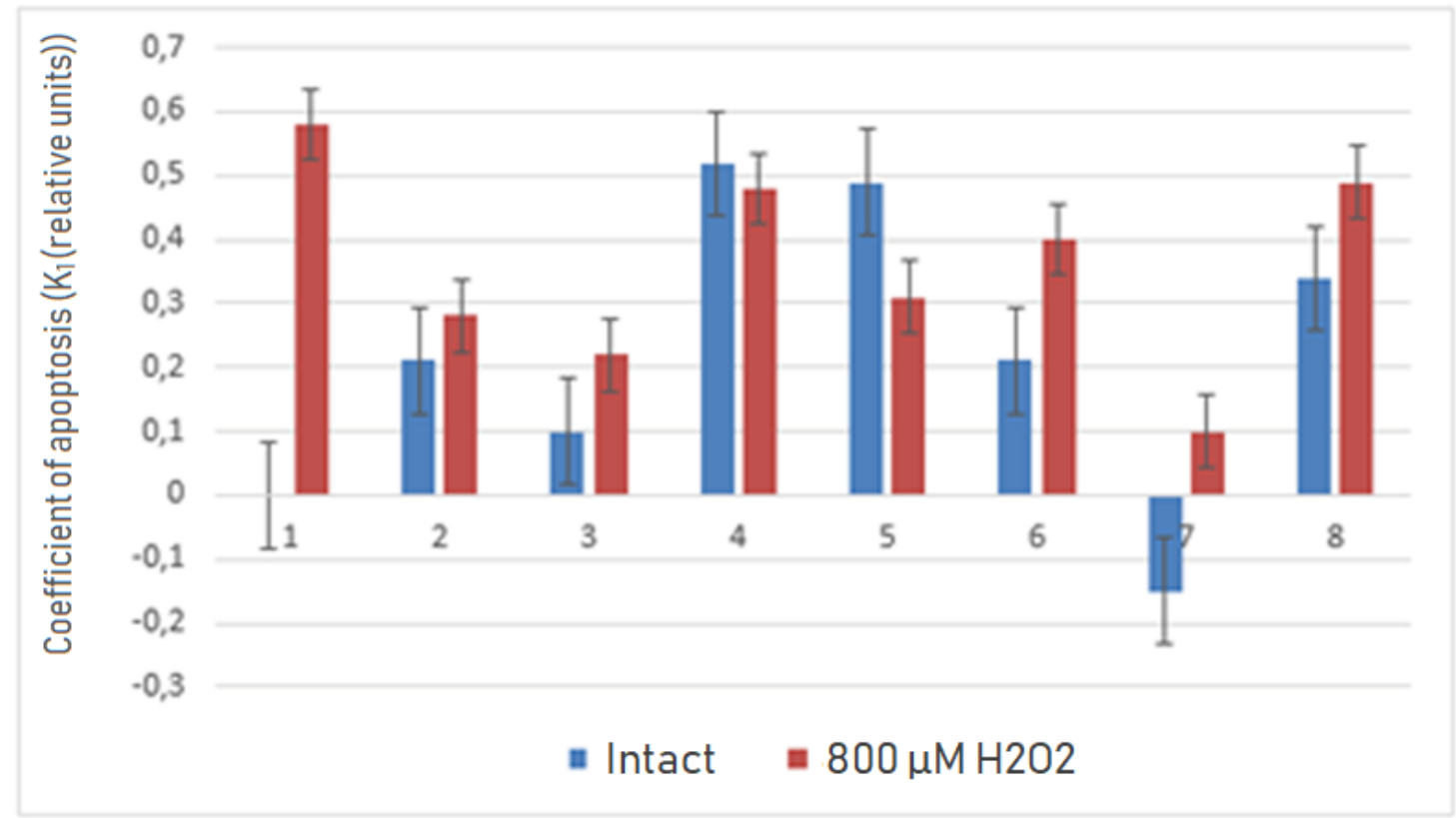

Figure 3. Coefficient of apoptosis $\left(\mathrm{K}_{1}\right)$ of MDCK cells under different incubation conditions (1MDCK cells, 2 - MDCK+beans "Tirkmela", 3 - MDCK + "Batumela-mindvris" beans, 4 - MDCK + beans "Udelebi", 5 - MDCK + beans "Shulavera", 6 - MDCK + green peas, 7 - MDCK + lentils, 8 - MDCK + soybean).

When different LCEs were added to Jurkat and MDCK cells incubated under oxidative stress conditions, the "Batumela-mindvris" beans' extract showed a pronounced cytoprotective effect on both cell types (reduced intensity of apoptosis by $23 \%$ and 35\%, respectively) while the extracts of "Tirkmela" and "Shulavera" beans and lentils reduced the intensity of apoptosis in MDCK cells (by $30 \%, 27 \%$ and $48 \%$, respectively).

Figure 4 presents the activity of antioxidant enzymes in intact and incubated Jurkat and MDCK cells under oxidative stress conditions. From the research results, it follows that the activity of antioxidant enzymes (SOD and catalase) in intact normal epithelial MDCK cells exceeds the value of these indicators in the leukemia-transformed Jurkat cells. At the same time, during the incubation of Jurkat cells under oxidative stress conditions, the activity of SOD and catalase changed insignificantly, whereas the activity of antioxidant enzymes sharply increased in MDCK cells (Figures 4A, B). These data indicate a significantly higher variability of enzymes in the antioxidant protection system of normal cells compared with tumor cells. 


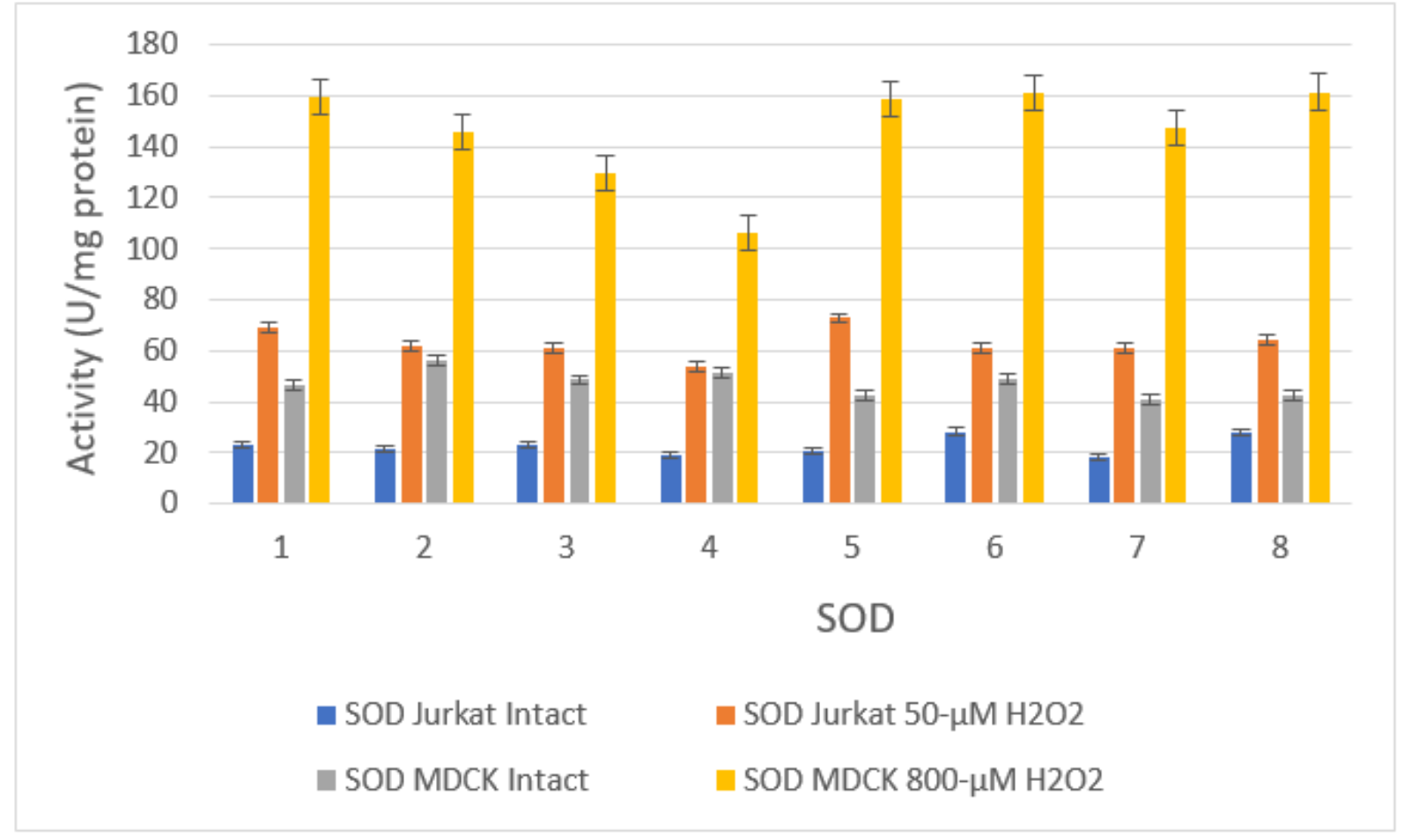

A

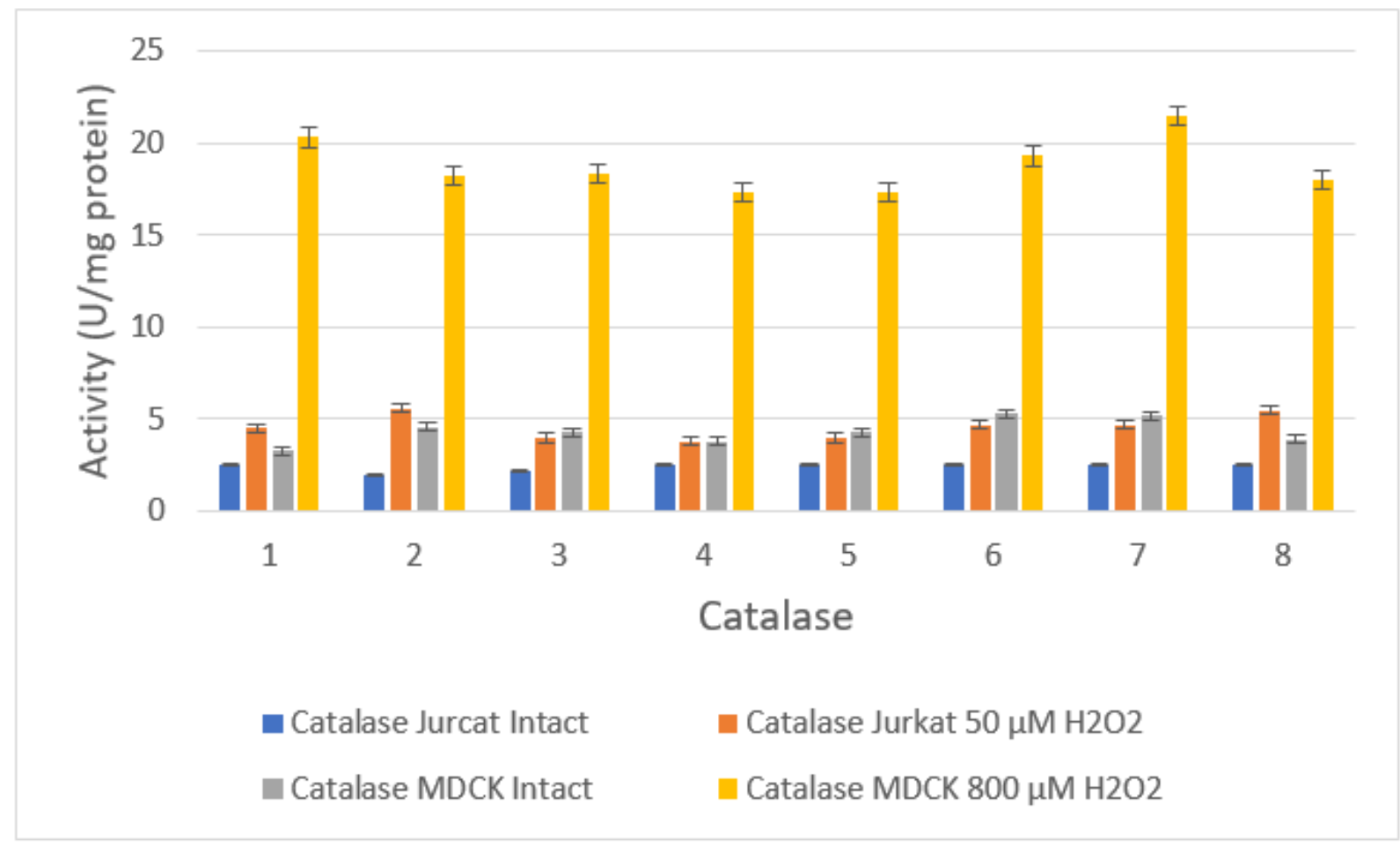

B

Figure 4. Antioxidant enzymes (SOD (A) and catalase (B)) activity of intact and incubated under oxidative stress conditions Jurkat and MDCK cells (1- intact cells, 2 - cells +beans "Tirkmela", 3 cells + beans "Batumela-mindvris", 4 - cells + beans "Udelebi", 5 - cells + beans "Shulavera", 6 cells + green peas, 7 - cells + lentils, 8 - cells + soybean). 
From the results of the study, it follows that the activity of antioxidant enzymes (SOD and catalase) in intact epithelial MDCK cells exceeds the value of these indicators in leukemia transformed Jurkat cells. At the same time, during the incubation of Jurkat cells under oxidative stress conditions, a slight alteration in SOD activity was detected, and catalase activity did not change at all, whereas in MDCK cells, the activity of these antioxidant enzymes increased dramatically (Figure 4A, 4B). These data indicate a significantly higher variability of the enzymatic antioxidant defense system of normal cells compared to those in tumor cells, and the data also explain why tumor cells have a particularly high sensitivity to oxidative stress $[19,20]$. Based on this, it is clear why we had to use significantly higher doses of hydrogen peroxide $\left(\mathrm{H}_{2} \mathrm{O}_{2}\right)(15$ times higher than in the case of Jurkat cells) in order to reduce the viability of MDCK cells by $20-30 \%$ in our experiments.

The results of current and previous studies $[15,21]$ have shown very different (cytoprotective and cytotoxic) effects of LCEs on intact and incubated under oxidative stress conditions Jurkat and MDCK cells. The correlation analysis indicates a direct dependence between the content of phenols and antiradical (antioxidant) activity (AA) of the studied extracts $(\boldsymbol{r}=0.79, \boldsymbol{p}=0.00002)$. These results prove the important role of the phenolic compounds in the mechanisms of the AA of LCEs.

In order to identify the cytoprotective and cytotoxic effects of LCEs on living organisms and to establish mechanisms of their activity, we conducted a correlation analysis of their anti-/proapoptotic effects on tumor Jurkat and normal epithelial MDCK cells with polyphenols content in extracts (and, consequently, their AA) and activity of the cellular enzymatic antioxidant defense system.

In the study of the cytoprotective/cytotoxic effectiveness of LCEs on the proliferative activity of intact Jurkat and MDCK cells, it was shown that the intensity of apoptosis in Jurkat and MDCK cells under the influence of various LCEs did not correlate with AA of these extracts (Jurkat: $\boldsymbol{r}=$ $0.018, \boldsymbol{p}=0.69$; MDCK: $\boldsymbol{r}=0,21, \boldsymbol{p}=0.75$ ). At the same time, no statistically significant correlation was found between the intensity of apoptosis and alterations in SOD and catalase activity in intact Jurkat and MDCK cells under the influence of the various LCEs (Jurkat: for catalase $\boldsymbol{r}=0.31, \boldsymbol{p}=$ 0.45 , for SOD $\boldsymbol{r}=0.28, \boldsymbol{p}=0.51$; MDCK: for catalase $\boldsymbol{r}=0.29, \boldsymbol{p}=0.49$, for SOD $\boldsymbol{r}=0.20, \boldsymbol{p}=0.63$ ).

These data suggest that the toxic effects of the LCEs on intact Jurkat and MDCK cells does not depend on extracts' AA (therefore, content of phenolic compounds) and redox-potential (activity of antioxidant enzymes) of the cells and indicate a different mechanism of extract toxicity. According to literature data, in addition to their antiradical activity, polyphenolic compounds also have ability to regulate the expression of proapoptotic proteins, Bax and Caspase-3 proteins [22].

Analysis of the relationship between the level of apoptosis in MDCK cells incubated under oxidative stress conditions and the content of phenols in LCEs added to the incubation medium of the cells indicates a statistically significant negative correlation between the cytoprotective effect of LCEs and the content of phenols in them $\left(r=-0.7759, \boldsymbol{p}=0.0236, \boldsymbol{r}^{2}=0.6020\right)$ (Figure 5A) (the higher the content of phenols in the extract, the lower the level of apoptosis). In the case of Jurkat 
cells, the causal relationship between the cytoprotective effect of LCEs extracts and their phenols content is broken (not statistically significant: $\boldsymbol{r}=-0.4096 ; \boldsymbol{p}=0.3135 ; \boldsymbol{r}^{2}=0.17$ ) (Figure 5B).

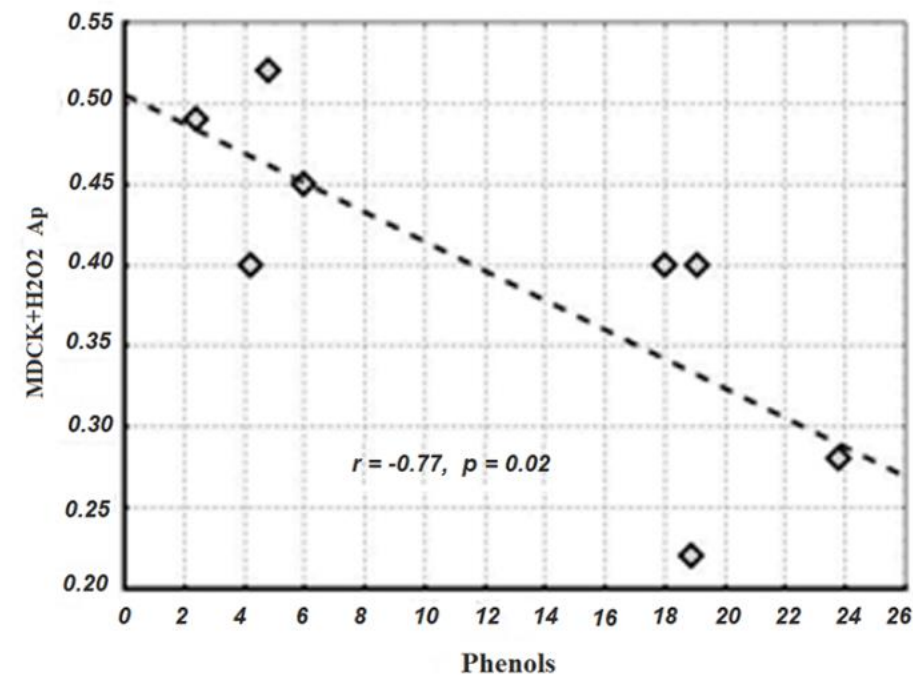

A

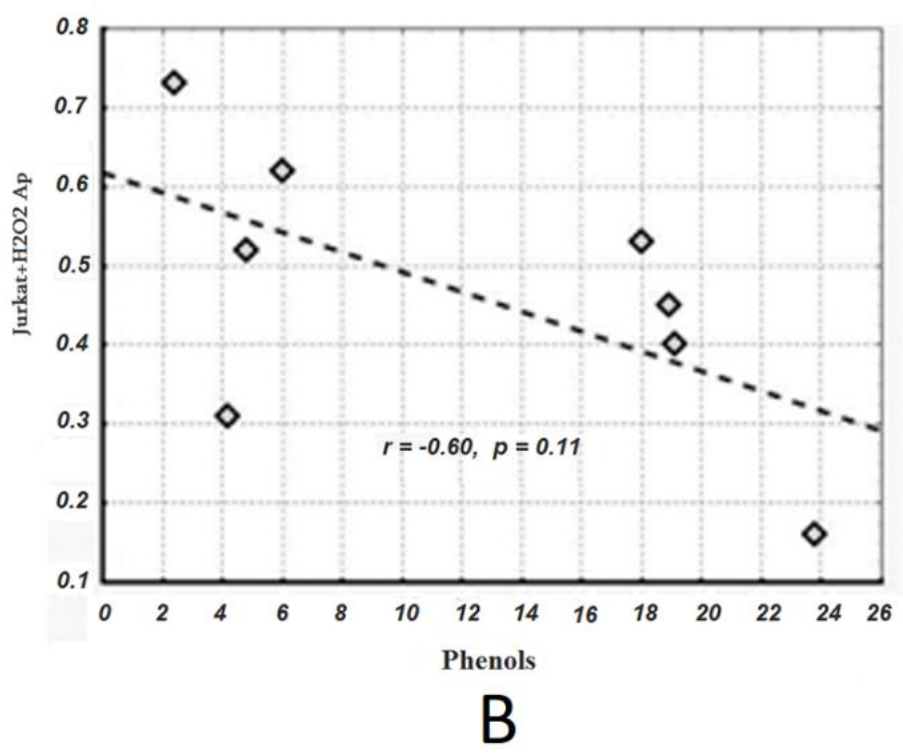

Figure 5. Correlation analysis of the relationship between the level of apoptosis in MDCK (A) and Jurkat (B) cells incubated under oxidative stress conditions and phenols content in LCEs added to the incubation medium of the cells (MDCK: $\boldsymbol{r}=-0.7759 ; \boldsymbol{p}=0.0236 ; \boldsymbol{r}^{2}=0.6020 ;$ Jurkat: $\boldsymbol{r}=$ $\left.0.4096 ; \boldsymbol{p}=0.3135 ; \boldsymbol{r}^{2}=0.17\right)$.

At the same time, a strong correlation $\left(\boldsymbol{p}=0.055, \boldsymbol{r}^{2}=0.50\right)$ was revealed between the level of apoptosis in intact and incubated MDCK cells under oxidative stress conditions and the influence of the various LCEs (Figure 6B); the high value of the determination coefficient $\left(r^{2}=0.50\right)$ argues in favor of a causal relationship ( $>50 \%$ ) between the effect of LCEs on the intact and incubated cells under oxidative stress. Indeed, the LCEs that showed the highest cytoprotective activity on the MDCK cells incubated under oxidative stress conditions ("Tirkmela" beans, "Batumela-mindvris" beans, lentils), had a weak cytotoxic effect on intact cells, or none at all, whereas cytotoxic for intact MDCK cells the "Udelebi," "Shulavera," and soybean extracts maintained high proapoptotic activity against MDCK cells incubated under oxidative conditions (Figure 3). These results and results of correlation analysis shown on the Figure 5A suggest that the cytoprotective (anti-apoptotic) activity of the LCEs in relation to normal epithelial MDCK cells is mainly due to their antiradical activity (high content of phenolic compounds).

In the case of Jurkat cells, no direct correlation was found between the protective effect of LCEs on intact cells and cells incubated under oxidative stress (statistically insignificant correlation: $\boldsymbol{p}=$ $0.30, r^{2}=0.009$ ) (Figure 6A). The results of our research show, however, that some of the LCEs that have a cytotoxic effect on intact Jurkat cells ("Udelebi" beans, soybeans) are also cytotoxic to cells incubated under oxidative stress conditions, but non-toxic for intact Jurkat cells extracts ("Tirkmela" beans) and slightly toxic ( "Sulavera" beans, green peas and lentils), contributed to increased apoptosis during incubation under oxidative stress conditions (Figure 2). Thus, it was impossible to establish an unambiguous picture of the cytoprotective/cytotoxic effects of LCEs on intact and incubated under oxidative stress conditions leukemia transformed Jurkat cells and the dependence of these effects on the phenols concentration in the extracts. 


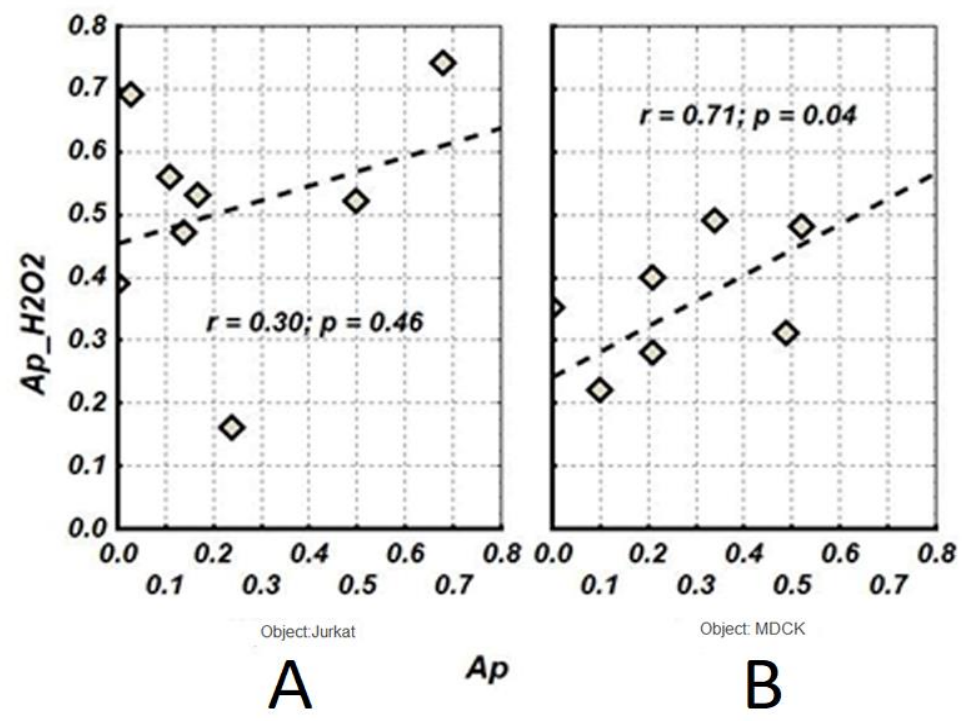

Figure 6. Correlation between the effectiveness of LCEs on intact and incubated under oxidative stress conditions MDCK (A) and Jurkat (B) cells (MDCK: $\boldsymbol{p}=\mathbf{0 , 0 5 5}, \boldsymbol{r}^{\mathbf{2}}=\mathbf{0 , 5 0}$; Jurkat: $\boldsymbol{p}=\boldsymbol{0 , 4 6 , \boldsymbol { r } ^ { 2 }}$ $=0,009)$.

Thus, it can be concluded that the cytoprotective activity of LCEs on normal epithelial MDCK cells incubated under oxidative stress conditions is related to their AA, whereas in the case of tumors Jurkat cells this redox-dependent cytoprotective mechanism is impaired.

Correlation analysis revealed a strong inverse dependence between the intensity of apoptosis and catalase (but not SOD) activity in intact MDCK cells incubated with various LCEs (catalase: $\boldsymbol{r}$ $=-0.6 . p=0.07$; SOD: $\boldsymbol{r}=0.04, \boldsymbol{p}=0.93$ ) (Figure 7A). In Jurkat cells, the presence of a weak directly proportional relationship between the intensity of apoptosis and catalase activity (catalase: $\boldsymbol{r}=0.3$, $\boldsymbol{p}=0.06)$ and the absence of a correlation between the intensity of apoptosis and activity for SOD (SOD: $\boldsymbol{r}=0.05, \boldsymbol{p}=0.90$ ) was detected (Figure 7B).

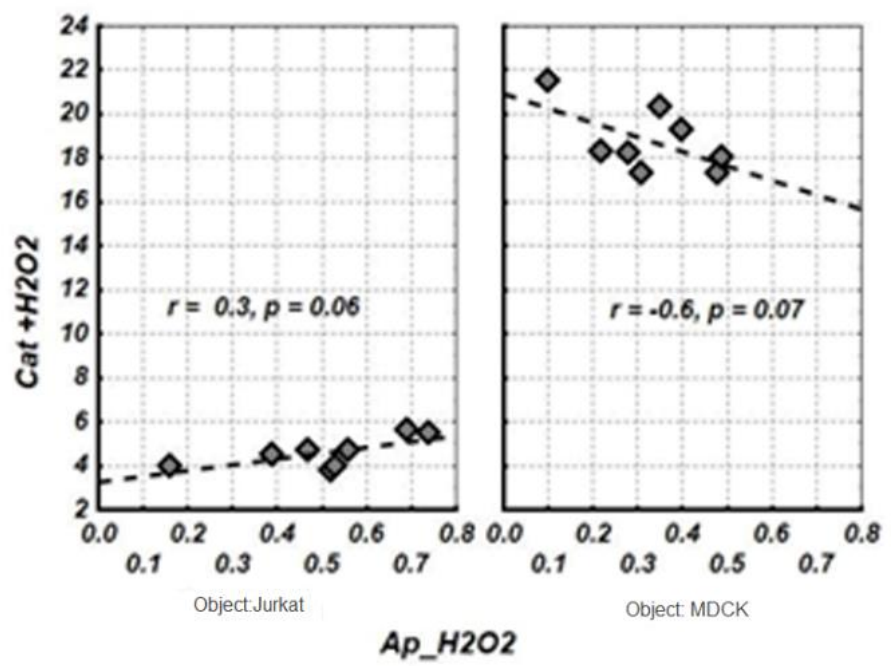

A

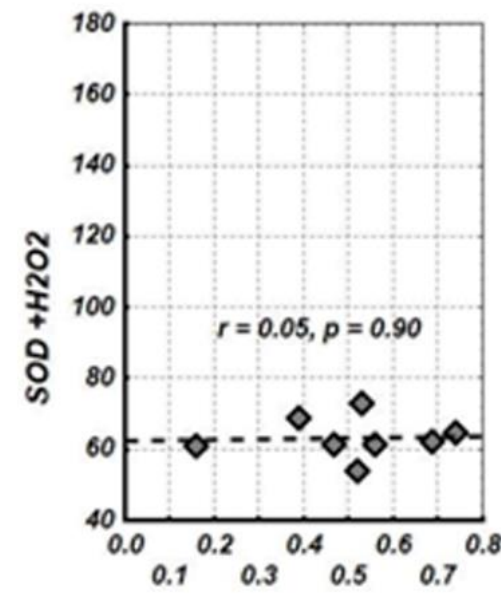

Object.Jurkat Ap_H2O2

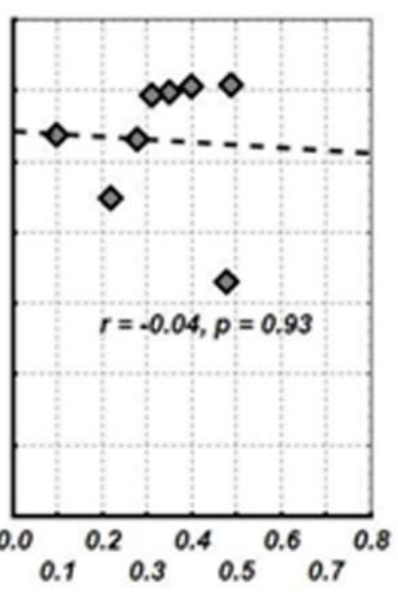

Object: MDCK

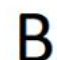

Figure 7. Correlation analysis of the dependence between apoptosis intensity in Jurkat and MDCK cells incubated in the oxidative stress conditions under the exposure of LCEs and the activity of the cellular enzymatic antioxidant defense system (SOD and catalase). 
These data support our assumption that the cytoprotective activity of LCEs in normal epithelial MDCK cells incubated under oxidative stress conditions is realized through redox mechanisms and that the phenol-rich extracts with a high AA, supplementing/stimulating the cells' antioxidant defense system, contributes to the stabilization of their proliferative activity (decrease in the intensity of apoptosis). It should also be noted that catalase plays a leading role in the redox mechanism of anti-apoptotic protection of MDCK cells.

In the case of leukemia transformed Jurkat cells, a similar redox-dependent mechanism for preventing apoptosis does not work, which is manifested by a lack of correlation between the intensity of apoptosis in Jurkat cells and AA of the LCEs and a weak positive correlation between the intensity of apoptosis of Jurkat cells and cellular catalase activity (extract-stimulated compensatory activation of catalase is insufficient to prevent apoptosis).

The important role of catalase in the mechanisms of anti-apoptotic protection of cells against oxidative stress is confirmed by the results of correlation analysis between intensity of apoptosis in incubated under oxidative stress conditions Jurkat and MDCK cells in the presence of LCEs and the initial activity of the antioxidant enzymes (SOD and catalase) in intact cells incubated with these extracts. The results of the correlation analysis (Figure 8) indicate the absence of any statistically significant relationship between the intensity of apoptosis and the enzymatic antioxidant enzymes (SOD, catalase) activity in Jurkat cells, and the presence of a strict statistically significant inverse correlation between the intensity of apoptosis and catalase (but not SOD) activity ( $\mathrm{r}=-0.72, \mathrm{p}=$ 0.045) in MDCK cells. These data indicate that, in the mechanism of the cytoprotective activity of polyphenol-rich LCEs on normal epithelial MDCK cells, along with their own AA, the stimulating effect of extracts on the catalase plays an important role on increasing the cellular antioxidant defense potential from the destructive effect of oxidative stress and preventing cell death. The presence of such mechanism in Jurkat cells has not been confirmed, which can be due to the low variability of the activity of enzymatic antioxidant defense system in tumor cell.

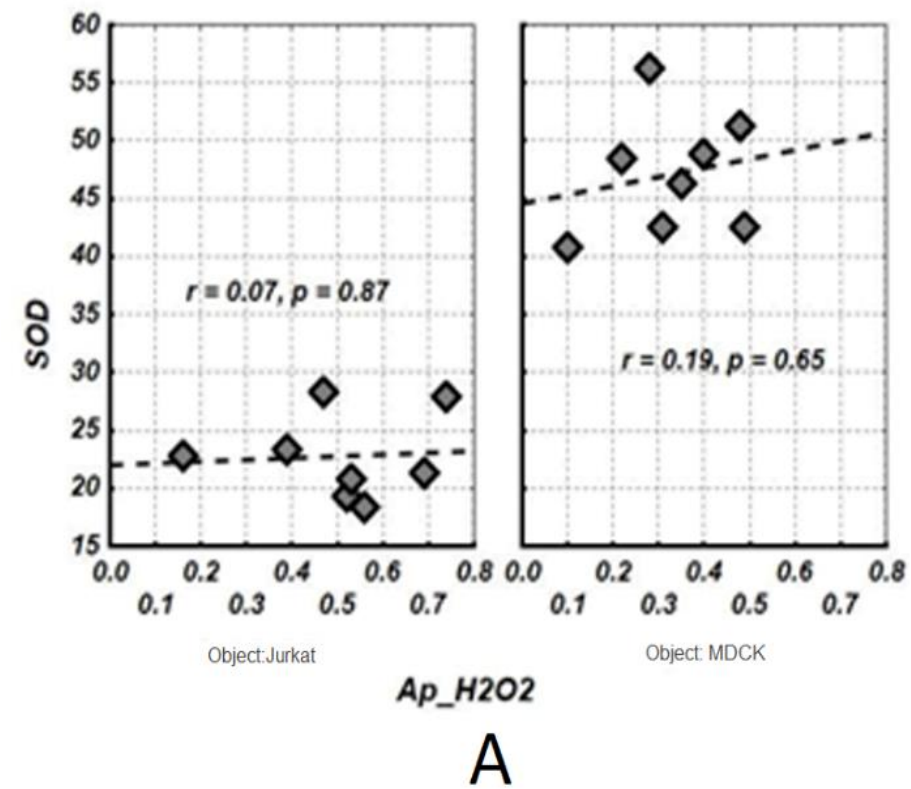

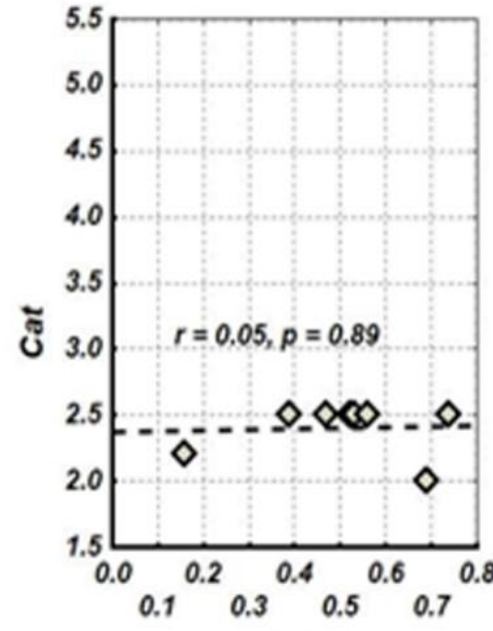

Object.Jurkat
Ap_H2O2

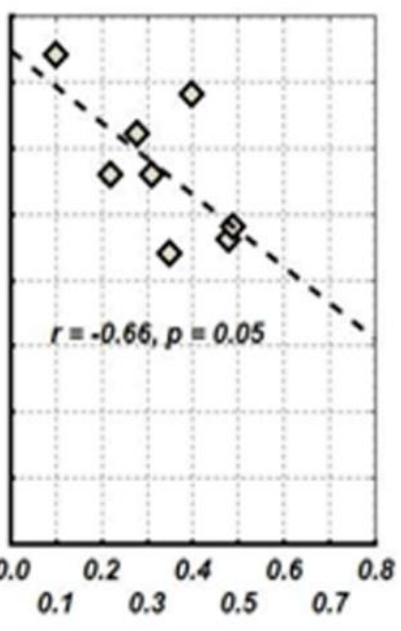

Object: MDCK

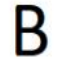

Figure 8. Correlation analysis of the dependence between the apoptosis intensity in the incubated in oxidative stress conditions Jurkat and MDCK cells under the exposure of LCEs and the initial activity of the cellular enzyme antioxidant system (SOD and catalase). 


\section{CONCLUSIONS}

Based on the analysis of the research results, it can be concluded that the differences in the effectiveness of various LCEs with different antiradical activity on intact and incubated under oxidative stress conditions Jurkat and MDCK cells may be partly associated with different initial antioxidant potential of these cells. The obtained result allow us to conclude that:

- The cytotoxic effect of LCEs on intact Jurkat and MDCK cells is independent of their antiradical activity and enzymatic cellular antioxidant defense system.

- The cytoprotective effect of LCEs on MDCK cells is realized through redox-dependent mechanisms and is associated both with the own antiradical activity of the extracts and with the stimulating effect of the extracts on the activity of enzymatic cellular antioxidant defense system.

- In the redox-dependent mechanism that prevents apoptosis in MDCK cells, catalase plays a leading role.

- LCEs did not protect Jurkat cells from oxidative stress-induced apoptosis through redoxindependent mechanisms that may be associated with low potential variability in the activity of the enzymatic cellular antioxidant defense system in tumor cells.

Analysis of the obtained results allow us to conclude that in cancer chemotherapy, the legume extracts might be combined with prooxidant drugs in order to protect normal cells, but not malignant ones, from their apoptosis-inducing effect. On the other hand, these extracts may protect nonmalignant tissues/organs from various apoptosis-related disorders.

List of Abbreviations: AIDS, acquired immune deficiency syndrome; MDCK, Madin-Darby Canine Kidney cells ; AA, antiradical activity; ROS, reactive oxygen species; ANOVA, Analysis of variance; SOD, antioxidant enzyme Superoxidedismutase; DPPH, 2,2-diphenyl-1-picrylhydrazyl; DSMZ, Deutshe Sammulung von Mikroorganismen und Zellkulturen; RPMI, Roswell Park Memorial Institute; FBS, fetal bovine serum; DMEM, Dulbecco's Modified Eagle Medium; PBS, phosphate buffer saline; MTT - 3-(4,5-dimethyltiazol -2)-2,5- diphenyl tetrazolium bromide; MTT - 3-(4,5-dimethyltiazol-2)-2,5-diphenyl tetrazolium bromide ; DMSO, Dimethyl sulfoxide,; SPSS, Statistical Package for the Social Sciences.

Competing interests: This study was conducted with research funds under contract with Ministry of Education, Science, Culture and Sport of Georgia. Under the "Tbilisi State Medical University Bioethics Committee Regulations," the research director and the investigators of this study declared necessary conflicts of interest to the ethical review committee.

Authors' Contributions: All authors contributed to this study.

Acknowledgments and Funding: We acknowledge the V. Bakhutashvili Institute of Medical Biotechnology of Tbilisi State Medical University Fund for funding, for access to the laboratory facilities and for guidance with cell culture. 


\section{REFERENCES}

1. Gottlieb RA, Nordberg J, Skowronski E, Babior BM: Apoptosis induced in Jurkat cells by several agents is preceded by intracellular acidification. Proc Natl Acad Sci USA 1996, 23; 93(2):654-8.

2. Peng YT, Chen P, Ouyang RY, Song L: Multifaceted role of prohibiting in cell survival and apoptosis. Apoptosis 2015, 20(9):1135-49.

3. Pérez-Garijo A, Steller H: Spreading the word: non-autonomous effects of apoptosis during development, regeneration and disease. Development 2015, 1;142(19):3253-62.

4. Curti V, Di Lorenzo A, Dacrema M, Xiao J, Nabavi SM, Daglia M: In vitro polyphenol effects on apoptosis: An update of literature data. Semin Cancer Biol 2017, 46:119-131.

5. Salucci S, Burattini S, Giordano FM, Lucarini S, Diamantini G, Falcieri E: Further Highlighting on the Prevention of Oxidative Damage by Polyphenol-Rich Wine Extracts. J Med Food 2017, 20(4):410-419.

6. Chkhikvishvili I, Sanikidze T, Gogia N, Enukidze M, Machavariani M, Kipiani N, et al.: Constituents of French Marigold (Tagetespatula L.) Flowers Protect Jurkat T-Cells against Oxidative Stress. Oxid Med Cell Longev 2016, 2016:4216285.

7. Chkhikvishvili I, Sanikidze T, Gogia N, Mchedlishvili T, Enukidze M, Machavariani M, et al.: Rosmarinic acid-rich extracts of summer savory (Saturejahortensis L.) protect Jurkat T cells against oxidative stress. Oxid Med Cell Longev 2013, 456253.

8. Davitashvili DT, Museridze DP, Svanidze IK, Gegenava LG, Sanikidze TV: Investigation of oxidative stress-induced alterations in the rat brain cortical cellular culture and their correction with vitamins E and C. Georgian Med News 2009, (177):73-7.

9. Davitashvili DT, Museridze DP, Svanidze IK, Pavliashvili NS, Sanikidze TV: Correction of oxidative stress in the rat brain cortical cellular culture with vitamines $\mathrm{E}$ and $\mathrm{C}$. Georgian Med News 2010, (180):56-60.

10. Enukidze MG, Machavariani MG, Intskirveli NA, Bezhitashvili ND, Sanikidze TV: Cell death in Jurkat cells induced by oxygen/nitrogen stress. Georgian Med News 2009, (167):109-13.

11. Lursmanashvili L, Gulua L, Turmanidze T, Enukidze M, Machavariani M, Sanikidze T: Biological activity of green tea extracts. Georgian Med News 2017, (263):88-93.

12. Urquiaga I, Avila F, Echeverria G, Perez D, Trejo S, Leighton F: A Chilean Berry Concentrate Protects against Postprandial Oxidative Stress and Increases Plasma Antioxidant Activity in Healthy Humans. Oxid Med Cell Longev 2017: 8361493.

13. Van Tits LJ, De Waart F, Hak-Lemmers HL, Van Heijst P, de Graaf J, Demacker PN, et al.: Effects of alpha-tocopherol on superoxide production and plasma intercellular adhesion molecule- 1 and antibodies to oxidized LDL in chronic smokers. Free Radic Biol Med 2001, 30(10):1122-9.

14. D'Angelo S, Martino E, Ilisso CP, Bagarolo ML, Porcelli M, Cacciapuoti G: Pro-oxidant and proapoptotic activity of polyphenol extract from Annurca apple and its underlying mechanisms in human breast c:ancer cells. Int J Oncol 2017, 51(3):939-948. 
15. Chkhikvishvili I, Maminaishvili T, Gogia N, Enukidze M, Machavariani M, Sanikidze T: Antioxidant, anti-inflammatory activity of Georgian leguminous crops cultures. Georgian Medical News 2017, 11 (272): 147-153.

16. Feng CQ, Ma WL, Song YB, Guo QY, Wu QH, Zheng WL: Detection of cell apoptosis by MTT assay. Di Yi Jun Yi Da Xue Xue Bao 2002, 22(3): 262-3.

17. Kuchukashvili Z, Burjanadze G, Menabde K, Chachua M, Dachanidze N, Mikadze M, et al.: Long-lasting stress, quantitative changes in nitric oxide concentration and functional state of brain mitochondria. Acta Neurobiol Exp 2012, 72: 40-50.

18. Tasset I, Pena J, Jimena I, Feijoo M, Del Carmen Munoz M, Montilla P, et al.: Effect of 17 beta-estradiol on olfactory bulbectomy-induced oxidative stress and behavioral changes in rats. Neuropsychiatr Dis Treat 2008, 4: 441-449.

19. Oberley LW: Antioxidant enzyme levels in cancer. Histol Histopathol 1997, 12: 525-535.

20. Sun Y, Oberley LW, Elwell JH, Sierra-Rivera E: Antioxidant enzyme activities in normal and transformed mouse liver cells. Int J Cancer 1989, 44: 1028-1033.

21. Mamniashvili TL, Chkhikvishvili ID, Enukidze MG, Machavariani MG, Kipiani Nana V, Sanikidze TV: Selective efficacy of Georgian legume extracts on Jurkat and MDCK cells. Georgian Medical News, 2019, 3 (288): 158-162.

22. Shih YZ, Huang AJ, Hou CY, Jiang CM, Wu MC: The stimulating effects of polyphenol and protein fractions from jelly fig (Ficusawkeotsang Makino) achenes against proliferation of leukemia cells. J Food Drug Anal 2017, 25(4):854-861. 